\title{
Transcatheter Patent Foramen Ovale Closure in a Child with Cryptogenic Ischemic Stroke
}

\author{
Eun Hye Lee ${ }^{1}\left(\mathbb{D} \cdot\right.$ Mi Young Han ${ }^{1}$
}

Received: 7 January 2020 / Accepted: 5 February 2020 / Published online: 11 February 2020

(C) Dr. K C Chaudhuri Foundation 2020

To the Editor: Patent foramen ovale (PFO) has been shown to be associated with stroke in adults, especially cryptogenic stroke, or stroke of undefined cause. There is an increasing evidence that paradoxical embolism through PFO may be the cause of cryptogenic stroke in children [1]. A 12-y-old girl presented with sudden onset of dizziness and body lateropulsion to the right side. She was unable to stand unassisted and could not stand or walk without falling to the right side. Diffusion weighted magnetic resonance imaging (MRI) of the brain showed an acute infarction in the left paramedian thalamus. A diagnosis of grade 3 PFO was made by agitated saline test. Brain magnetic resonance angiography and thorough laboratory evaluation to determine the cause of the ischemic stroke failed, so we presumed that paradoxical emboli could be the primary cause of stroke in this patient and decided to close the PFO by interventional cardiac catheterization. Our decision was supported by the very low risk of PFO closure compared with the relatively high risk of lifelong anticoagulation in children and adolescents. There were no complications, and a postprocedural transthoracic echocardiogram showed appropriate device location without residual shunt. The antiplatelet agent was discontinued after 4 mo, and there has been no recurrence of stroke during $3.5 \mathrm{y}$ of follow-up.

Recent expert review suggested that PFO closure may be considered in selected children with cryptogenic stroke and a significant right-to-left shunt with no other risk factors [2]. Recent adult studies indicate that PFO closure with a device is superior to medical therapy for reducing the risk of stroke recurrence in cases of stroke associated with PFO [3]. A longterm follow-up study also demonstrates that PFO closure is

Eun Hye Lee

leeeh80@gmail.com

1 Department of Pediatrics, College of Medicine, Kyung Hee University Hospital, Seoul, South Korea associated with a lower rate of recurrence of ischemic stroke than medical therapy and showed good safety [4].

We suggest that it is worth considering PFO closure in children and adolescents based on recent ongoing studies in adults. The procedure also makes it possible to discontinue long-term antithrombotic treatment, which is difficult to maintain and has a number of possible side-effects, including bleeding.

\section{Compliance with Ethical Standards}

Conflict of Interest None.

Source of Funding This study is supported by the Basic Science Research Program of the National Research Foundation of Korea funded by the Ministry of Science, ICT and Future Planning (NRF2017R1C1B5076772).

\section{References}

1. Bartz PJ, Cetta F, Cabalka AK, et al. Paradoxical emboli in children and young adults: role of atrial septal defect and patent foramen ovale device closure. Mayo Clin Proc. 2006;81:615-8.

2. Eleftheriou D, Ganesan V. Controversies in childhood arterial ischemic stroke and cerebral venous sinus thrombosis. Exp Rev Cardiovasc Ther. 2009;7:853-61.

3. Abdelaziz HK, Saad M, Abuomara HZ, et al. Long-term outcomes of patent foramen ovale closure or medical therapy after cryptogenic stroke: a meta-analysis of randomized trials. Catheter Cardiovasc Interv. 2018;92:176-86.

4. Saver JL, Carroll JD, Thaler DE, et al. Long-term outcomes of patent foramen ovale closure or medical therapy after stroke. New Eng J Med. 2017;377:1022-32.

Publisher's Note Springer Nature remains neutral with regard to jurisdictional claims in published maps and institutional affiliations. 